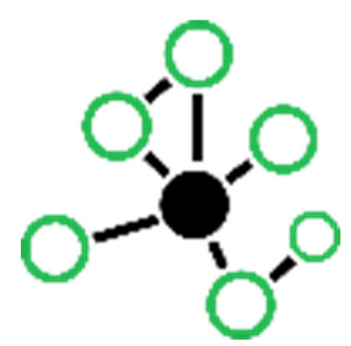

\title{
Fair Data Points on standardised Plant data within WUR
}

\author{
Hendrickx, P. ${ }^{1}$ \\ ${ }^{1}$ Wageningen University \& Research, Droevendaalsesteeg 1, 6708PB Wageningen, the Netherlands \\ Corresponding author's e-mail: patrick.hendrickx@wur.nl
}

Within Wageningen UR there are many datasets available. The Centre for Genetic Resources Netherlands (CGN) has a well curated collection of different crops. It catalogs the background information about their accessions. Plant Breeding has phenotypic information about those crops. Both databases are independent and not integrated. Integration of both datasets saves time for researchers but also gives extra possibilities.

In this project we produced Fair Data Points for the crop tomato derived from the CGN and the Plant Breeding database using the best standards available. We created a visual interface which makes it possible to ask questions accessing both datasets using our fair datapoints.

This illustrates the power of standarisation data for making them better accessible and reusable. 